\title{
INVESTIGACIONES RECIENTES SOBRE ETNOCIENCIA EN EL AREA MAYA
}

\author{
ROBERTO EscaLANTE \\ Lingüistica, INAH
}

Al contemplar los últimos diez años, salta a la vista el avance en la comprensión de ia etnociencia no sólo en el área maya; podemos mencionar tres o cuatro tesis de maestría de la E.N.A.H. (Prestán, 1975; Ramirez 1975; Fragoso, 1978; Cuevas, 1979), los proyectos de medicina popular y psiquiatría transcultural y etnobotánica de varias instituciones I.N.A.H., U.N.A.M., I.P.N., IMEPLAN, Dirección General de Culturas Populares, U.N.A.M., Universidad Veracruzana, INN, ENAH, etc.); aunque entre algunos colegas todavía se le considera como "ciencia ficción" o como estudios subjetivos. Sin embargo, es triste ver lo poco que hacen los antropólogos mexicanos en este campo, y que todavía tenemos que recurrir a las investigaciones de EEUU.

Si se considera el objetivo de la etnociencia como "la presentación de modelos más o menos formales (algebraicos) de la estruc. tura cognoscitiva de aspectos delimitados del comportamiento hu. mano" (Day, 1973) podemos entender el énfasis científico de estos estudios y el desarrollo teórico-metodológico actual. Pero nunca se debe perder de vista que se intenta aprehender otra realidad psico. lógica, otra visión del mundo de la cual no pueden participar fácilmente el antropólogo, el biólogo o el médico, como en la etiología del susto (Collado, 1974) donde solamente se hace una traducción terminológica de la curación del espanto pero sin entender los postulados conceptuales y de orden ideológico que hacen satisfactoria esta curación.

Los biólogos hablan de sistemas cognoscitivos populares y entre los antropólogos todavia se discute si debe ser etnociencia o ciencia 
folk (Hunn, 1977), arguyendo que la etnociencia es una rama de la antropología que se preocupa por los conceptos y sus regularidades distribucionales como el contenido esencial de la cultura, en tanto que el estudio de la ciencia folk trata de entender el papel del hombre en la naturaleza. Como tal puede ser incluida en ci campo más inclusivo de la Antropología cognoscitiva ya que ésta se preocupa no sólo del conocimiento del hombre de su ambiente físico, sino también de su conceptualización de la realidad social.

Al hablar de la zoología folk de los tzeltales de Tenejapa, Hunn (op. cit.), la define como "el análisis descriptivo de lo que es conocido dentro de una comunidad particular acerca de la clasificación, morfologia y comportamiento animal, $y$ al estudio comparativo de tales sistemas de conocimiento". Distingue este mismo autor tres procesos cognoscitivos que interactúan en la definición de los sistemas biológicos folk (¿podriamos decir folklóricos o populares?), que son: la formación y adquisición de conceptos que lleva a su organización en un sistema clasificatorio; el proceso semántico que lleva a cabo la tesignación de categorías y la noción intuitiva de taxonomía. Según Kay (1971) esto involucra una estructura taxonómica de las categorias, un conjunto de nombres (lexemas) y una proyección que los relaciona con las categorias; el tercer proceso es el desarrollo de características configuracionales por el cual se identifican las categorias.

La investigación de la ciencia folk comienza con la obtención de conjuntos léxicos exhaustivos de un campo específico (sea éste la botánica, la zoologia, la anatomía, etc.), pero al mismo tiempo tra. tando de evitar la respuesta puramente conceptual de listas de palabras, como se verá en la investigación del color, y tratar de definir las categorías nombradas, y luego instar a los informantes a que ordenen estas categorias para que finalmente surja la estructura semántica. Estas relaciones pueden tener varias formas de organización como el paradigma, intersección de varios rasgos; la taxonomía que presenta relaciones de jerarquía y contraste; relaciones del todo a las partes como en los sistemas anatómicos, etc.

Berlin (U. of California) en conjunción con Breedlove (California Academy of Sciences) y Raven (Missouri Botanical Garden) ha propuesto los principios generales de clasificación y nomenclatura de la biologia folk (1973):

1) En todas las lenguas es posible aislar lingüisticamente grupos reconocibles de organismos, de varios grados de inclusión, Estas clases se llaman taxa (plural de taxon) y pueden ser ejemplificados 
por grupos de organismos, indicados por los nombres cedro, bejuco, planta, pájaro carpintero, etc., en español.

2) Los taxa se agrupan en categorias taxonómicas etnobiológicas, definibles en términos de criterios lingüisticos y taxonómicos y que son seis: iniciadora singular (unique beginner), forma de vida, gené rica, específica y varietal. También ha propuesto la categoría intermedia.

3) Estas cinco categorías son ordenadas jerárquicamente y los taxa asignados a cada rango son mutuamente exclusivos, excepto por la iniciadora singular de la cual sólo hay un miembro.

4) Los taxa de la misma categoría etnobiológica se hallan en el mismo nivel taxonómico, dentro de cualquier estructura taxonómica particular. El taxon que es miembro de la categoria iniciadora sin. gular se halla en el nivel cero. Los taxa de forma de vida se hallan sólo en el nivel uno. Los taxa genéricos se hallan de manera característica en el nivel dos, pero si no, aparecen en el nivel uno. Los taxa específicos se hallan característicamente en el nivel tres, pero de lo contrario en el nivel dos y están inmediatamente incluidos en un taxon genérico que aparece en el nivel uno. Los taxa varietales, si están presentes, característicamente se hallan en el nivel cuatro, pero si no, en el nivel tres.

$\mathrm{Y}$ representa estas relaciones entre categorias taxonómicas etnobiológicas y sus niveles taxonómicos relativos en un diagrama (Fig. 1). También observa las siguientes tendencias generales:

5) El taxon que se halla en la categoria más inclusiva, iniciadora singular, raramente es nombrado por una expresión simple, p. cj. planta, animal.

6) Los taxa que son miembros de la categoria "forma de vida" son muy pocos y van de 5 a 10 (auque puede haber sistemas que contradigan este principio de Berlin et al., donde no haya más de dos formas de vida, como sucede en la zoología folk tzeltal, donde sólo hay mut 'aves' y chambalam 'animales'). Todos los taxa de esta categoría son politípicos, es decir, que se dividen en taxas de jerarquía inferior. Están designados por lexemas primarios (la tipología de lexemas se explica más adelante) como árbol, bejuco, pájaro, pasto, mamifero, etc.

7) En las taxonomías folk típicas los taxa que son miembros de la categoría etnobiológica "genérica" son mucho más numerosos que las formas de vida, llevando hasta 500 miembros y son desig. nados por lexemas primarios. Por ejemplo, pino, ced,o, gorrión, etc.

Los taxa genéricos son las piedras miliares de todas las taxo- 
nomías folklóricas. Representan los grupos de organismos más comúnmente referidos en el ambiente natural, son los más sobresalientes psicológicamente y tal vez sean los primeros taxa que aprenden los níños.

8) Los taxa que son miembros de las categorias "especifica" y "varietal" son menos numerosos que los taxa genéricos. Ambos son designados por lexemas secundarios. Ejemplos de taxa específicos: rosa de Castilla, gato montés. Ejemplos de taxa varietales: maiz pinto, gato de Angora.

9) Los taxa intermedios son aquellos que se incluyen en las formas de vida y que incluyen taxa del rango genérico. Son muy raros en las taxonomias y cuando se puede comprobar su existencia no están designados lingüisticamente, por lo que les considera categorias no manifiestas (Covert).

En otra publicación, Berlin (1972) propone el siguiente orden evolutivo de las seis categorias lingüisticas:

genérica $\rightarrow\left\{\begin{array}{l}\text { forma de vida } \\ \text { específica }\end{array} \rightarrow \quad\left\{\begin{array}{l}\text { intermedia } \\ \text { varietal }\end{array}\right\}\right.$ iniciadora singular

Esto significa que primero apareció la categoria genérica, en seguida la categoría especifica o la forma de vida: luego, en el siguiente paso, la categoria varietal o la intermedia y finalmente la categoria singular; esto para la historia de cualquier idioma.

En su tipologia de lexemas, Berlin et al. (1917) establecen un criterio distribucional para clasificarlos (como se ve en los principios anteriormente expuestos), asi distinguen entre lexemas primarios y lexemas secundarios. Los lexemas primarios pueden ser noranalizables cuando están formados por un solo morfema, p. ej. rosa, Los lexemas primarios formados por dos o más moriemas pueden ser de dos tipos: analizables improductivos, cuando se trata de construcciones semánticas exocéntricas, diente de león, nombre de una hierba, que no se reficre a ninguna clase de diente o de león o pueden ser analizables productivos, cuando son construcciones semáticas endocéntricas como vibora de cascabel, que se refiere a una víbora, y se considera primario porque contrasta en el mismo nivel taxonómico con lexemas de un morfema como cobra o boa. Los lexemas secundarios también están formados por dos o más morfemas en una construcción semántica endocéntrica, como rosa de Borbón, que es una clase de rosa, pero se le considera secundario porque contrasta, en el mismo nivel taxonómico con otros lexemas de dos o 
más morfemas, como rosa de los Alpes, o rosa de Castilla y nunca con lexemas de un solo morfema.

Hunn, en su Tzeltal folk zoology (1977), propone la nomenclatura ideal isomórfica como una proyección de uno-a-uno del corpus de nombres al conjunto de taxa, de modo que cada taxon tenga un nombre único y que cada nombre invariablemente se refiera a un solo taxon. Sin embargo, hay cinco desviaciones de este ideal:

1) Sinonimia. Se desvia del ideal ya que un solo taxon es designado por más de un nombre. En tzeltal el gato doméstico, se le conoce con tres nombres:

xawin que es el nombre real; mir que tiene connotaciones obscenas y kátu, que se reconoce como préstamo del español.

2) Homonimia, Que se desvia porque hay designaciones idénticas que se refieren a mís de un taxon, $y$ los nombres idénticos tienen distintas etimologías, como en español baya color y baya vaina.

3) Polisemia. Esta desviación ocurre cuando las varias aplicaciones de un nombre demuestran relaciones semánticas. Puede ser de dos tipos:

1. Los dos conceptos pueden estar relacionados por inclusión de conjuntos: En tzeltal mut designa pájaro y gallina.

2. Los dos conceptos pueden no estar relacionados: tzeltal chan serpiente y clase de escarabajos.

4) Categorías no manifiestas (Covert). Se desvian del ideal ya que un taxon cuya existencia es demostrable, no lleva designación alguna. En treltal, los ratones del género Peromyscus, se reconocen como un grupo pero que no lleva designación.

5) Nombres sueltos. Este tipo de desviaciones son designaciones reconocidas sin una denotación muy clara ni atributos definidos. Por ejemplo, en tzeltal $x$ 'ain aunque designa 'cocodrilo' algunos informantes aplicaron este nombre a la ballena, que nunca habian visto, porque era también grande y acuática.

Se han hecho las siguientes normalizaciones: ch:čjx:s̆, šs; 't?; ts:c; vv: v. ; i:ä; h:j; j:x. 
Nivel 0

Nivel $1 \quad \mathrm{fv}_{1} \quad \mathrm{fv}_{2} \ldots f \mathrm{fv}_{\mathrm{n}}$

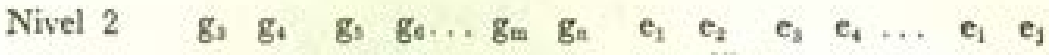
e: específica

Nivel $3 e_{2} e_{3} e_{2} e_{4} \ldots \quad e_{m} e_{n} \quad$ IS: Iniciadora singular

fv: forma de vida

Nivel $4 \quad v_{1} v_{2} \quad v_{m} \quad v_{n} g$ : genérica

e: especifica

Figura 1. Diagrama de categorias taxonómicas etnobiológicas y sus niveles taxonómicos relativos. (Berlin et al., 1973).

La categorización del color en Mesoamérica es un importante estudio que inició Robert McLaury en 1979 y que será la base de su tesis de doctorado en la Universidad de California, Berkeley. venque no podemos presentar aquí sus resultados finales o su interpretación, es interesante resumir el método de trabajo de campo, por las implicaciones que tiene en el campo de la etnosemántica. El diseño del equipo y algunos de los métodos provienen directa. mente del proyecto de B. Berlin y P. Kay, "Categorización humana del color". El material consiste de 330 fichas de matices de colores Munsell, acomodadas al azar con respecto al espectro y numeradas consecutivamente y una tabla del espectro.

La obtención de los datos se logra mostrándole al informante 330 fichas de matices de colores Munsell, acomodadas al azar con respecto al espectro. De las respuestas lingüisticas, se eligen los términos diferentes, o sea los lexemas nucleares no modificados, y se le pide al informante que señale, en el espectro, el punto o puntos de color que represente el mejor ejemplo de cada lexema; estas selecciones son los puntos focales de sus categorías de color. El siguiente paso es identificar los morfemas libres y ligados, de las respuestas, incluyendo los lexemas nucleares que hacen referencia exclusivamente al color, palabras para objetos naturales y todos los modificadores, afijos, reduplicadores, etc. Después se vacian los da. tos obtenidos al azar a una gráfica del espectro y luego se colorea según las categorias de color de la lengua y esto mostrará las ca. tegorías básicas, los limites de categorías, las regiones focales, las categorias secundarias, etc.

Además de la obtención de formas lingüisticas, se trata de ob- 
tener datos etnosemánticos: ¿cuáles palabras son absolutamente esenciales para la proyección léxica del espectro y cuáles designan divisiones secundarias o subcategorias? Se determina la inclusión de categorías y la extensión de cada categoria de color en el espectro. Las respuestas de esta etapa podrán revelar que algunas glosas son semánticamente sinónimas, obteniendo datos cognescitivos que en muchos casos revelan un estadio de categorización anterior a los datos linguíisticos, como ha sucedido en algunas lenguas mayas.

La "Etnociencia de los grupos lingüisticos del Golfo de México" (Esca:ante, 1980) abarca idiomas de tres grupos: mixe-zoque (mixe, mixe-popoluca, zoque-popoluca), nahua (Mecayapan y Pajapan) y maya (chontal de Tabasco). Del chontal se han tomado ejemplos de clasificadores numerales que se citan más adelante; los ejemplos de anatomia incluyen, aparte del chontal (chh.), ejemplos del mixepopoluca (mixe-pop.) de esta misma investigación.

Aunque es imposible presentar aquí las clasificaciones de plantas (Berlin et al., 1974) y de animales (Hunn, 1977) en total, daremos una idea de cómo se ajustan estas obras monumentales a la teoria que hemos descrito. Ambas proceden de la comunidad tzeital de Tenejapa.

En Principles of Tzeltal plant classification, dicen los autores que los tzeltales conciben el reino vegetal más o menos en los mismos términos que la sistemática botánica occidental; sin embargo, el dominio como clase conceptual no está marcado por ninguna expresión lingüística comparable al término español planta (cf. principio 5). También hay muchas expresiones que pueden ser utilizadas para contrastar cualquier miembro del reino vegetal con un miembro de otro reino, p.ej. animal. Asi las plantas 'no se mueven' ma $x$ nihik; 'no caminan' ma xberik, y los animales si. En terrenos lingüísticos más formales, los nombres de plantas se hallan con el clasificador numeral tehk. Los clasificadores numerales son expresiones obligatorias que deben ser usadas al contar ciertos objetos en tzeltal como en otras lenguas del área maỹ (véase más adelante). Así, 'tres árboles' deberia ser expresado oxtehk te 'tres-miembros de la clase de plantas'árboles'; los nombres de animales, por otra parte, $s e$ hallan con el clasificador numeral koht y los nombres para seres humanos se hallan con el clasificador tul.

En el nivel de las formas de vida, la botánica tzeltal presenta cuatro taxa: $t e$ ' árboles; $a k$ bejucos; ' $a k$ pastos y wamal hierbas. En el nivel genérico se presentan 471 taxa, de los cuales 356 , o sea un $75 \%$ se incluyen dentro de las formas de vida. Cerca de 97 for- 
mas genéricas (como un 20\%) no se incluyen en ninguna de las cuatro formas de vida, y son consideradas por los tzeltales como géneros no afiliados, este tipo de plantas, son, sin excepción, cultivadas o morfológicamente peculiares; p.ej. 'ixim maiz, chenek frijol, halal bambú y chi maguey. Finalmente aproximadamente $5 \%$ son ambiguos ya que presentan categorías de dos (o rara vez tres) clases de formas de vida. De los 471 taxa genéricos, 91 son designados por préstamos, principalmente del español. Los 380 restantes son designados for expresiones tzeltales nativas que pueden analizarse como lexemas primarios. De éstos, 101 son lexemas primarios simples, 125 son designados por lexemas primarios improductivos y 154 son designados por lexemas primarios productivos. A continuación se citan algunos ejemplos:

Primarios simples

'on aguacate (Persea americana)

'ich chile (Capsicum pubescens)

Primarios improductivos

tsis chaok ruda de la pradera (Thalictrum Guatemalense)

(pedo-trueno)

balam K'in girasol silvestre (Polymnia maculata)

(jaguar-dia)

Primarios productivos

kul'ak clase de zarzaparrilla (Smilax spp.)

(NO-bejuco)

chitam'ak clase de pasto (Muhlenbergia macroura) (cerdo-pasto)

La mayoría de los taxa genéricos en la etnosistemática tzeltal son monotipos (esto es, no tienen mayor división); sólo 73 taxa son politípicos, incluyendo de 2 a 15 clases especificas. Existen 237 taxa específicos que, con algunas excepciones, son designados por lexemas secundarios, por ejemplo:

Nombres genéricos
st'ul
amaranto

(Amaranthus spp.)

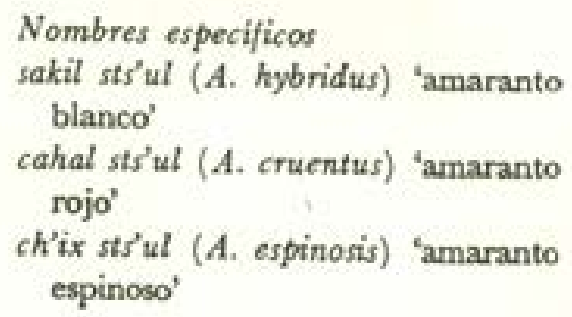


Hay casos en que los taxa específicos pueden ser designados por lexemas primarios, como es el caso de los tipos específicos, donde uno de los taxa es referido sólo por el nombre genérico (por el uso polisémico del nombre genérico). Por ejemplo:

Nombre genérico
k'ewex

anona
Nombres especificos

k'ewex (Anona cherimola) 'anona (tipo)'

ch'isch'is k'ewex (A. muricata) 'anona espinosa'

k'etoex max (A. reticulata) 'anona de mono'

Aparte de capítulos dedicados a la nomenclatura, a la morfología de las plantas y a su uso, el capítulo 6 dedicado a la flora estudia todos los taxa. Cada párrafo comprende el nombre binario latino para establecer la correspondencia con el nombre vernículo, la descripción cientifica indigena, los usos, las plantas relacionadas, y cuando es posible los criterios de identificación, y un diagrama que muestra la estructura taxonómica tzeltal interna, además del número de colectas de cada especie. En el plano lingüistico, cada nombre es analizado morfológicamente $y$ se indican sus equivalentes en español $\mathrm{e}$ inglés cuando existen. El libro concluye con indices cruzados de gran utilidad. También cuenta con gran cantidad de fotografias y mapas.

Concebido de la misma manera, el libro de Hunn (1977), sigue el mismo plan y presentación que la etnobotánica tzeltal y los dos son indispensables para los estudiosos de la ciencia folk y altamente característicos del nuevo énfasis en la ciencia popular. Ambos in. cluyen además sendos capítulos sobre la ecología y la etnografia de Tenejapa; y además postulados teórico-metodológicos que son básicos para cualquier estudio de la etnobiología.

Al pasar a la zoología folk tzeltal, nos sorprende que algunos de los principios generales de Berlin no se cumplen en este campo, En el nivel más alto de la taxonomía está el término chanbalam animal, lit. chan serpiente, etc, y balam jaguar. El hombre se excluye de esta clasificación porque los animales "no hablan como la gente, y porque viven en la selva: no tienen almas".

En el nivel de las formas de vida, extrañamente y contradicien. do el principo 6, sólo se hayan dos formas de vida designadas por lexemas primarios: mut aves y chanbalam mamífero (uso polisémico). 
Los taxa genéricos, agrupados dentro de estas dos formas de vida y dentro de grupos complejos, no manifiestos o nombrados, suman 335 , de los cuales 106 son de la categoría mut ave, y 45 de la categoría chanbalam mamiferos, y finalmente 184 que no se incluyen en ninguna forma de vida. La mayoría de los taxa genéricos son monotípicos, 280 o sea $84 \%$. Los 55 restantes $(16 \%)$ incluyen 2, 3 o más divisiones.

Las proporciones relativas de los nombres de los taxa genéricos difieren notablemente de los nombres genéricos de plantas (véase arriba). Por ejemplo, $51 \%$ de los nombres animales genéricos son lexernas primarios simples y sólo $27 \%$ de los nombres de plantas son de esta clase. Una explicación puede ser los préstamos, otra los nombres onomatopéyicos.

Lexemas primarios simples:

ch'o rata, ratón; pach' pato doméstico; chikitorox hormiga soldado.

Lexemas primarios improductivos:

1. Nombres pseudoespecíficos:

Yuxtaposición de dos nombres animales:

K'ulub kawudyu 'chapulin poltrón'

(langosta caballo)

Término de pseudo-estado de vida.

yal ha 'pupa de mosca'

(su hijo mosca)

2. Analogia descriptiva.

mayil ti'bal 'armadillo'

(calabaza-carne)

3. Madre de —; Yuxtaposición ambiental o relación protectiva. $m e^{\prime} k$ 'ulub 'gavilán de cola blanca'

(madre-langosta)

4. Construcciones agentivas.

hse' te' 'rata trepadora'

(corta-ramas)

5. Construcciones puramente descriptivas. sak hol 'tayra, cabeza de viejo' (blanca-cabeza)

6. Complejos onomatopéyicos. chi'chi' bulbul 'reyezuelo doméstico' (dulce-dulce-espuma-espuma) 
Formas compuestas. Incluyen lexemas primarios analizables y lexemas secundarios ya que son sintácticamente equivalentes.

\section{COLOR}

Los cinco colores tzeltales aparecen en los nombres de animales: tsah rojo; tsahal wirin papamoscas.

'ihk' negro; 'ihk'al ts'unun colibrí

$k^{\prime} a$ amarillo; $k^{\prime}$ an toht petirrojo

sak blanco; sakil sanich hormiga

yax verde; yax hex grajo

\section{TAMANO}

ch'in chico; ch'in honon abejorro

muk' grande; muk'ul pay zorrillo

\section{PATRON}

barsin barcino, esp.; barsin chan escarabajo barcino

\section{TEXTURA}

tsots pelo; tsots 'it ha mosca con pelos en la panza

$c h$ 'ix espina; $c h^{\prime} i x$ 'uhchum puerco-espin (espina-tlacuache)

FORMA. (Metafórico).

chan culebra; chan tsay anguila (culebra-pez)

Esta obra incluye también un análisis de la anatomía animal, de los animales aprovechados en la caceria y en la medicina. El capitulo 5 de la fauna, incluye la descripción detallada de todos los taxa, con el diagrama de sus relaciones taxonómicas. Las entradas para los taxa genéricos y especificos incluyen el nombre tzeltal y su etimologia, después una definición en términos de los taxa científicos. Sigue una breve relación descriptiva de los organismos comprendidos, su morfología, comportamiento y distribución. Se enlistan los nombres "primarios" y las variantes sinónimas. Se incluyen datos de interés etnográfico, de especies comestibles, su categoria de $K^{\prime}$ ixin caliente o sikil frío, su uso en la medicina.

\section{CLASIFICADORES}

Como se mencionó en la discusión de la botánica tzeltal, los clasificadores que acompañan a los numerales, en la mayoria de las lenguas de ta familia maya, son un primer índice en la clasificación de las cosas, en yucateco -fuul clasifica personas y animales; -peel 
objetos, sin especificar su forma, y -kuul, para árboles y plantas; en chontal -tek, clasifica plantas y árboles; en lacandón -tuts'u', sirve para contar rios. Pero también tienen la función de clasificar las formas, así en yucateco; ts'üt, sirve para contar objetos largos y delgados; -waal, objetos planos y pequeños; -wool, objetos redondos; -heek', gajos. Una función muy importante es que estos clasificadores sirven como designadores de medida, peso y volumen, en Yuc. -saap, para contar brazas; -naab, palmos; -luub leguas; en itzá -kaap, cuartas; en quiché $-x a^{\prime}$, pasos. También sirven para contar el tiempo: lacandón $t$ 's'it, lunas; en yucateco -suutuk, un momento; -haab, años. $\mathrm{Y}$ también para contar números, en yucateco -lot pares; en chontal $-k^{\prime} a$, grupos de cinco; y en quiché, -much', ochenta; -lahuh, del 11 al $14 ;-$ thuh, del 15 al 19 ; -winak, $y-k^{\prime} a l$ veinte. $\mathrm{Y}$ muchos otros en otras lenguas.

\section{ANATOMfA}

El conocimiento tradicional de la anatomia tiene, entre los ma. yas, como en cualquier otro grupo humano, niveles de conocimiento que dependen de la especialización, el cazador y el carnicero sabrán más sobre la anatomía animal, mientras que el curandero y el huesero conocen más del cuerpo humano, pero entre el hablante medio, la partonomía es uno de los campos semánticos que se adquieren a temprana edad. La partonomía exige también su nomencla. tura y aquí también se hace el análisis lexémico como en la etnobotánica; se observa si los términos son polisémicos como en el chontal $\mathrm{Ki}$ mano y brazo, y cuando esto sucede determinar los puntos focales y su extensión; en el tzeltal 'akan pierna-pie y $k$ 'ab brazomano, en el chontal 'ok pie y pierna. Este fenómeno no sucede en huasteco okob brazo, $k$ 'ubak mano; pero si en akan, pierna-pie.

Este tipo de polisemia no es exclusivo de las lenguas de la familia maya, ya que lo vemos también en mixe-popoluca, $k r^{\prime}$ manobrazo, tan, pie-pierna, y en matlatzinca $y \dot{k}$ mano-brazo, mó pie-pierna. La nomenclatura puede ser con un lexema simple, como en estos casos, pero también se puede recurrir a construcciones endocéntricas como en huasteco pulek okob biceps (gran brazo) pulek akan muslo (gran pierna). O también se recurre a metáforas, en huasteco mim in k'ubak pulgar (señora de la mano), sukul in akan pantorrilla (estómago de la pierna). En tzeltal se observan los siguientes compuestos: 
bak' scmilla, en sbak' yat testiculo (semilla del pene), sbok' sit pupila (semilla del ojo);

$n u k^{\prime}$ cuello, en snuk' sk'ab muñeca (cuello de la mano), snuk' yakan tobillo (cuello del pie): chnluk'u-ok be camino, en sbe xchux uretra (camino de la orina), sbe sim fosa nasal (camino de moco), sbe yik' tráquea (camino del aire).

Esta lista no es exhaustiva, se citan sólo algunos ejemplos.

En chontal se observan las siguientes construcciones:

$\begin{array}{ll}\text { tsuts pelo, en } & \begin{array}{l}\text { tsuts-hut pestaña (pelo-ojo) } \\ \text { tsuts-'at vello (pelo pene), } \\ \text { tsuts-tuch vello (pelo-vulva) }\end{array}\end{array}$

pich piel, en

pich-hut párpado (piel-ojo)

pich-ti' labio (piel-boca)

püch-pan cuero cabelludo (piel-cabeza)

$t a^{\prime}$ excremento en $a^{\prime}$-hut lagaña (excremento-ojo)

ta'ni moco (excremento-nariz)

ta'chikin cerilla (excremento-oido)

$n i^{\prime}$ nariz, en $n i^{\prime}-k i^{\prime}$ dedo (nariz-mano), cf. mixe-pop.

ki-hihp

$n i^{\prime}$-ok dedo del pie (nariz-pie), cf. mixe-pop

tan-hihp

$n i^{3}$-tun glande (nariz-pene)

$n i^{\prime} c h u^{\prime}$ pezón (nariz-seno), cf. tzeltal sn'xchu'

Otro dominio del conocimiento tradicional, hasta ahora poco explotado, que ha sido estudiado a profundidad entre los lacandones por Bruce (1975) es la interpretación de los sueños. Esto tiene repercusión no sólo en la psicología transcultural 'sino hasta en la parapsicología, pues en el primer tomo el autor cita algunos sueños interpretados por Chan $K^{\prime}$ in de Najá y su confirmación posterior.

En general, la interpretación onírica lacandona se basa en tres principios, comparables a la interpretación freudiana:

1. ba'ik u tus, 'una clase de mentira', "reverso", según Freud.

a. el hombre simboliza un animal, por medio del onen, clasificación simbólica.

b. inversión de cualidades, cantidades y atributos. 
2. hach u pixan, 'el alma verdadera de la persona o entidad'. Según Freud, la representación directa.

3. $u$ k'in, 'su profecia' la metáfora de Freud: representación del todo por la parte o un objeto asociado.

De los 1000 sueños registrados corresponden sólo 55 profecías, de éstas sólo 12 envuelven el $90 \%$ de los símbolos oníricos.

1. Sangre: Se sueña con achiote, con color rojo o yax, verde.

2. Muerte: Se sueña con armadillo o animal en su cueva. Temblor.

3. Fin del mundo: Incensario.

4. Miel: Atole o bebidas del maíz.

5. Enfermedad: Lagartijas: escalofrío. Fuego: fiebre.

6. Jaguar: Rocas, piedras, obsidiana.

7. Carne o presa, o venado (onen) : Ropa. Conquista.

8. Mono, deidades solares del onen mono.

9. Lluvia y fenómenos meteorológicos: Lágrimas y luto, Hormigas.

10. Serpientes: Cañas, flautas, cuerdas, trenza, puro, pene, etc.

11. Visitantes, a través del onen.

12. Jabali deidades del onen de la tierra.

Este tipo de clasificaciones se llaman simbólicas, ya que no se basan en la morfología, como se ve en los apodos de animales, en los dias del calendario maya con nombres de animales.

Para terminar consideremos tres clases de clasificaciones no-biológicas pero que tienen que ver con el universo o con el comportamiento humano. Una es la del comportamiento verbal en tzotzil (Bricker, 1973); otra es la clasificación humana en jacalteco (Day, 1973) y la jerarquía de la comida quiché (Hanson, 1975). 
Subgrupos del comportamiento verbal en tzotzil (Chamula).

Discurso

Formal

$\left(-k^{\prime} o p\right)$

Discurso

Informal

(-lo'il)
Oraciones

K'opon

k'op riox

Contemplación

K'opoh 'o'on

Planeación

koplal

Guerra

$k^{\prime} \circ p$

Debate

kop

Crítica

'ut bail

$\left\{\begin{array}{l}\text { Difamación } \\ \text { Regaño }\end{array}\right.$

Charla frívola

loko lo'il

'ixtol to'il

'ixtol k'op

Chisme

lo'iltabe

Ridiculo

labanvaneh

Discusión

lo'ilta 
Clasificadores nominales para personas en jacalteco.

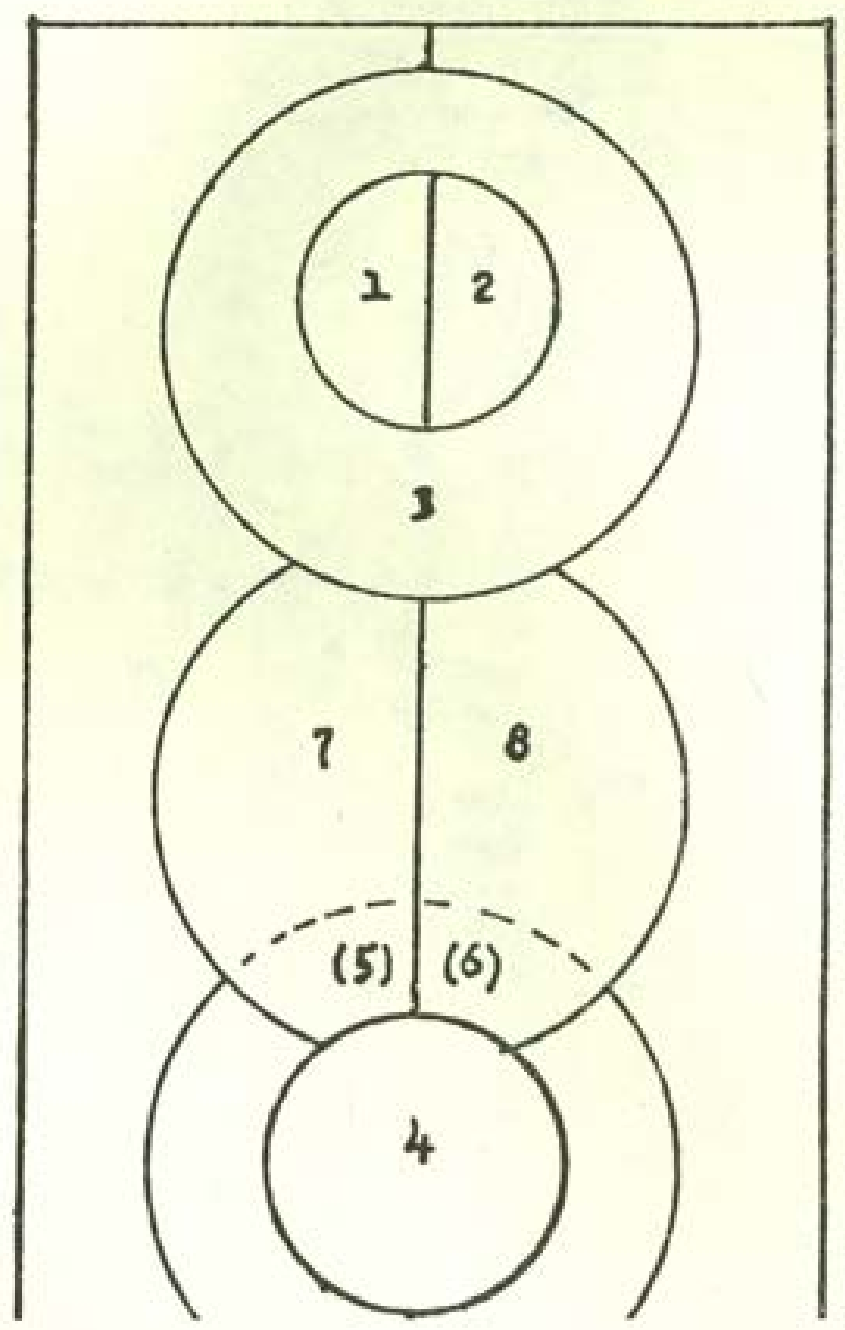

1,2. koman, komi' 'deidad: masculina, femenina'

3. $y a$ ' 'no-deidad respetada'

4. 'unin 'criatura'

5,6. ho' ni'an $x 0^{\prime}$ ni'an 'niño pariente no-criatura: masc., fem.

7.8. ho', $x o^{\prime}$ 'pariente no criatura no respetado: masc., fem.

9,10. naj ni'an, 'ix ni'an 'niño no-criatura no pariente: masc, fem.

11.12. naj ix 'no niñe, no pariente, no respetado: masc., fem. 
Jerarquía de la comida chiqué.

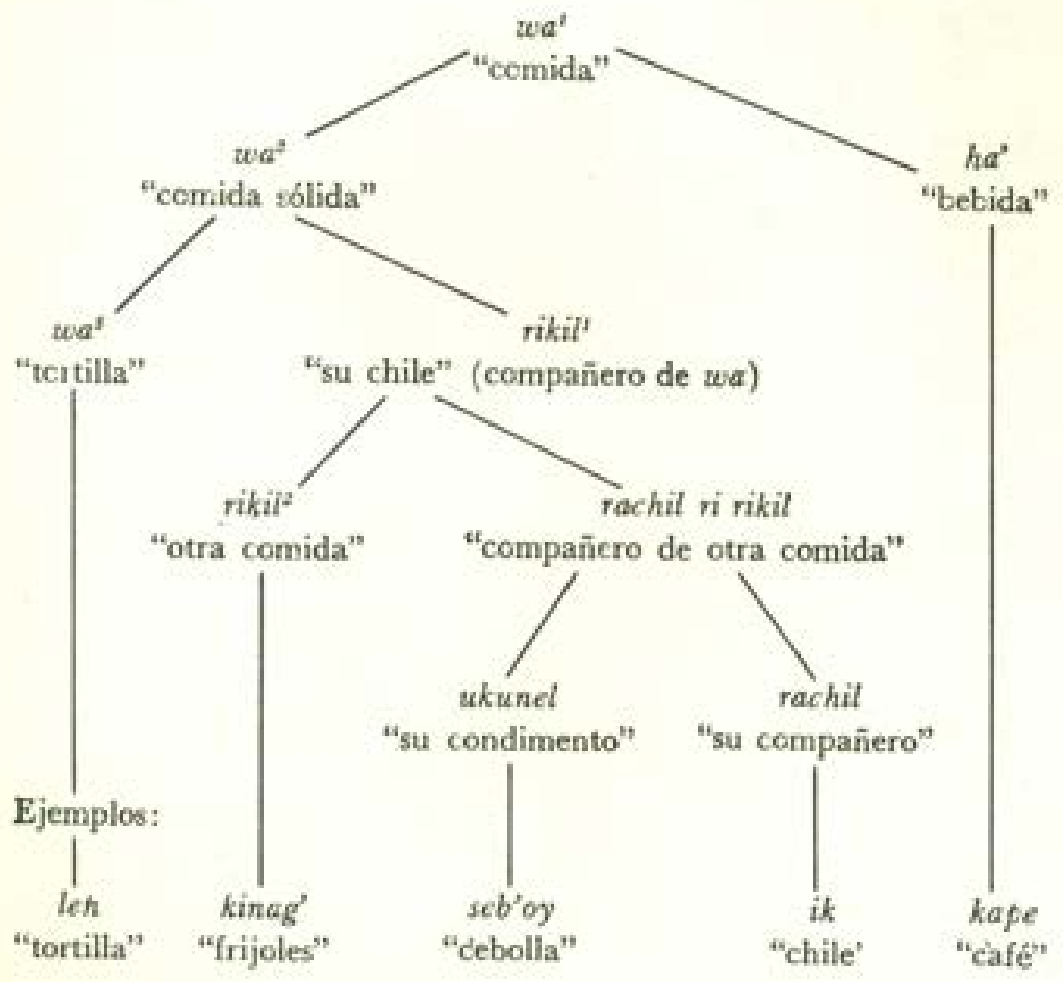




\section{REFERENCIAS}

Beruin, B.

1972 Speculations on the Growth of Ethnobotanical Nomenclature, Language in Society 1, 61-98.

D. E. Brentove y P. H. Raven

1973 "General Principles of classification and nomenclature in folk biology", American Anthropologist, 75, 214-42.

1974 Principles of Tzeltal Plant Classification, New York, Academic Press.

Besckxk, V. R.

1973 "Three genres of Tzotzil insult", Meaning in Mayan Langwages, edited by Munro Edmonson, 183-204, The Hague: Mounton.

Brown, C. H.

Bruce, R. D.

Date from Huartec (Northern Veracruz), Ms.

1968 Gramática del lacandón, Depto, de Inq. Antrop. INAH, Pub. no. 21, México.

1975 Lacandon Dream Symbolism, Ediciones Euroamericanas, México.

Campaena, LyLi

1977 Bibliography of Mayan languages and Linguistics, Institute for Mesoamerican Studies.

Collando, A., R. (UNAM)

1974 "Etiología y teraptutica del susto en una cornunidad zapoteca", American Antropological Association, Reunión anual 73a. Mexico.

Cuevas, S.

1979 Ornitologia Amuzga, Tesis de Maestría en Lingüistica, ENAH.

Dsy C.

1973 "The semantics of social categories in a transformational grammar of Jacaltec", Meaning in Mayan Languages, edited

Escalantz, R. by Munro Edmonson, La Haya, Mouton, 85-105.

1974 Datos etnosemánticos del nocabulario matletzined, XLI Congreto Internacional de Americanistas, México.

1980 Etnociencia de los grupos lingüisticos del Golfo de Merico, Ms.

Fracoso, R.

1978 La etnomedicina matlatzince, Teșis de Maestria en Antropología Social, ENAH.

Hunson de Henne, M.

1975 "La comida quiché: Su estructura cognoscitiva en Chichicas. tenango, Guatemala", XIV Mesa Redonda de la Sociedad

HUNN, E, S. Mexicana de Antropologia, Tegucigalpa.

1977 Tzeltal Folk Zoology, Academic Press, New York.

KAY, $P$.

1971 "On Taxonomy and semantic contrast", Language 47: 866887.

MeClaurr, $\mathbf{R}$.

1980 Linguistic color eategorization in Mesosmerica: Instructions for deseriptive field work, Ms. 
Pexstix, A.

1975 El wo de la chicha y la sociedad cuna, III, Ediciones Especiales 72 .

Roserao C., M.

1961 Morfemas clasificadores del Maya yucateco, a William Townsend, pp. 657-662, México.

Scutumann G. O.

1971 Descripción estructural del maya itzd del Petén, Guatemala, C.A., Centro de Estudios Mayas, UNAM, México, Cuaderno 6. 